\title{
Histoplasmose Cutânea Disseminada em Doente com SIDA: Relato de um Caso Clínico
}

\author{
A. Kombo', I. Monteiro', N. César' , G. Catorze², M. Henriques', R. Manuel', A. Cunha' ', G. Luciano' \\ 'Serviço de Dermatologia do Hospital Central de Maputo, Maputo, Moçambique \\ 2Serviço de Dermatologia do Hospital de Egas Moniz, Lisboa, Portugal
}

RESUMO - Descreve-se o caso clínico duma mulher de 30 anos, raça negra, natural e residente em Moçambique, internada no serviço de dermatologia do Hospital Central de Maputo por múltiplas pápulas de aparecimento progressivo e aditivo disseminadas por todo o tegumento cutâneo. O teste para VIH 2 foi positivo, com CD4 de $1 \mathrm{cel} / \mathrm{mm}^{3}$. O exame histológico de uma das lesões foi compatível com infecção por Histoplasma capsulatum. Foi medicada com anfotericina B durante 21 dias com melhoria clínica. Teve alta medicada com fluconazol $400 \mathrm{mg} /$ dia e tratamento anti-retroviral mas viria a falecer 13 meses depois após gastrenterite aguda.

PALAVRAS-CHAVE - Dermatomicoses; Infecções Oportunistas Relacionadas com a SIDA; Histoplasmose; Síndrome da Imunodeficiência Adquirida.

\section{Cutaneous Histoplasmosis in AIDS Patient: Case Report}

ABSTRACT - This report describes a case of disseminated cutaneous histoplasmosis in a 30 years old black woman, born and living in Mozambique, with disseminated eruptive multiple papules, some of them with erosions, localized also in palms, plants and oropharyngeal mucosa. The HIV 2 test was positive and the CD4 count was $1 \mathrm{cel} / \mathrm{mm} 3$. Histopathology was compatible with infection by Histoplasma capsulatum. She was treated with amphotericin B during 21 days, with clinical improvement, followed by oral fluconazole $400 \mathrm{mg} /$ day and antiretroviral, but she died 13 months later from gastroenteritis.

KEYWORDS - Acquired Immunodeficiency Syndrome; AIDS-Related Opportunistic Infections; Dermatomycoses; Histoplasmosis.

\section{INTRODUÇÃO}

A histoplasmose é uma micose sistémica causada por: a) Histoplasma capsulatum var. capsulatum com distribuição mundial; b) histoplasma capsulatum var duboisii, própria do continente africano. ${ }^{1} O$ habitat natural deste fungo é o solo contaminado por fezes de aves e morcegos. ${ }^{2}$ A doença é amplamente distribuída no continente Americano e Africano, e é rara na Europa à excepção da Itália. ${ }^{1,3}$ Com o advento da SIDA o número de casos de histoplasmose tende a aumentar, principalmente na sua forma disseminada.

A doença transmite-se por inalação de esporos suspensos na atmosfera de cavernas, capoeiras e construções veIhas alcançando os alvéolos pulmonar e disseminam para outros órgãos por via linfática e hematogénica. A inoculação cutânea directa é rara. Os indivíduos imunodeprimidos são mais susceptíveis à doença. As lesões cutâneas são polimorfas: pápulas, nódulos, com aspecto moluscóide ou variceliforme e úlceras que curam deixando manchas híper ou hipopigmentadas. Pode afectar as mucosas. O diagnóstico faz-se por cultura de fluidos corporais ou tecidos. A histopatologia mostra um processo granulomatoso supurativo, com necrose e infiltrado inflamatório linfocitário e neutrofílico. O tratamento de eleição é anfotericina $B$ desoxicolato.

Reportamos um caso de histoplasmose cutânea sem envolvimento de outros órgãos em doente com SIDA.
Correspondência: $M^{a}$ Goreti Catorze

Serviço de Dermatologia

Hospital Egas Moniz

R. da Junqueira 126

1349-019 Lisboa, Portugal

DOI: https://dx.doi.org/10.29021/spdv.76.1.801
Recebido/Received

30 Agosto/August 2017

Aceite/Accepted

14 Dezembro/December 2017 


\section{Caso Clínico}
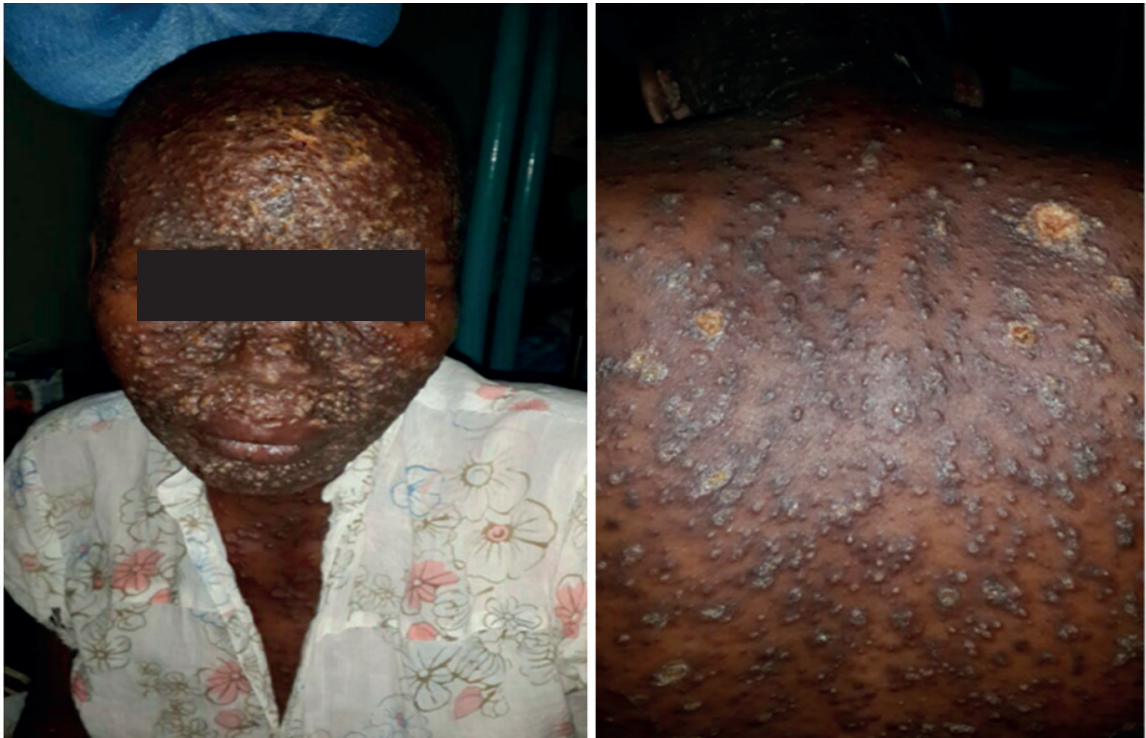

Figura 1 e 2 - Aspecto clínico das lesões papulosas disseminadas.

\section{RELATO DE CASO CLÍNICO}

Mulher de 30 anos de idade, raça negra, natural e residente em Moçambique, doméstica, internada no serviço de Dermatologia do Hospital Central de Maputo em 2014 por múltiplas pápulas de aparecimento progressivo e aditivo, algumas erosionadas, disseminadas por todo o tegumento cutâneo incluindo as palmas, plantas e mucosa orofaríngea. Tratava-se duma dermatose extensa deixando poucas áreas de pele sã (Fig.s 1, 2, 3 e 4), sem adenomegálias. A doença teve início 3 meses antes do internamento. Na semana anterior estas queixas associaram-se a febre, odinofagia e mal-estar geral. O teste para VIH 2 foi positivo, com CD4 de $1 \mathrm{cel} / \mathrm{mm}^{3}$.
Foi realizada biópsia cutânea de uma das lesões cujo exame histopatológico foi compatível com histoplasmose (Fig.s 5 e 6): derme com infiltrado inflamatório granulomatoso com numerosos histiócitos contendo no seu interior estruturas arredondadas pequenas translúcidas sugestivas de corresponderem a Histoplasma capsulatum (colorações de hematoxilina-eosina e Grocott). Não foi possível medir a dimensão destas estruturas pelo que é impossível apontarmos para a variante de histoplasma e não foi feita cultura para exame micológico. A pesquisa de BAAR (bacilos álcool-ácido-resistentes) negativa.

A radiografia de tórax foi normal, não foi realizada radiografia do esqueleto ósseo. As análises do sangue
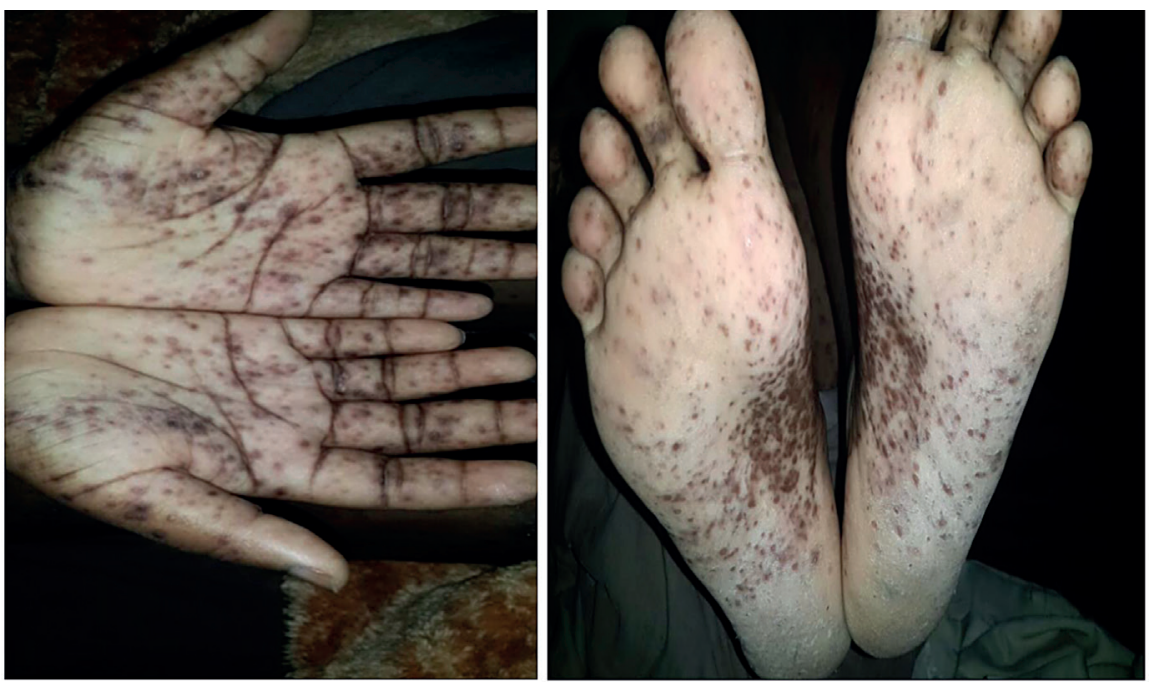

Figura 3 e 4 - Aspecto clínico das lesões papulosas disseminadas. 


\section{Caso Clínico}

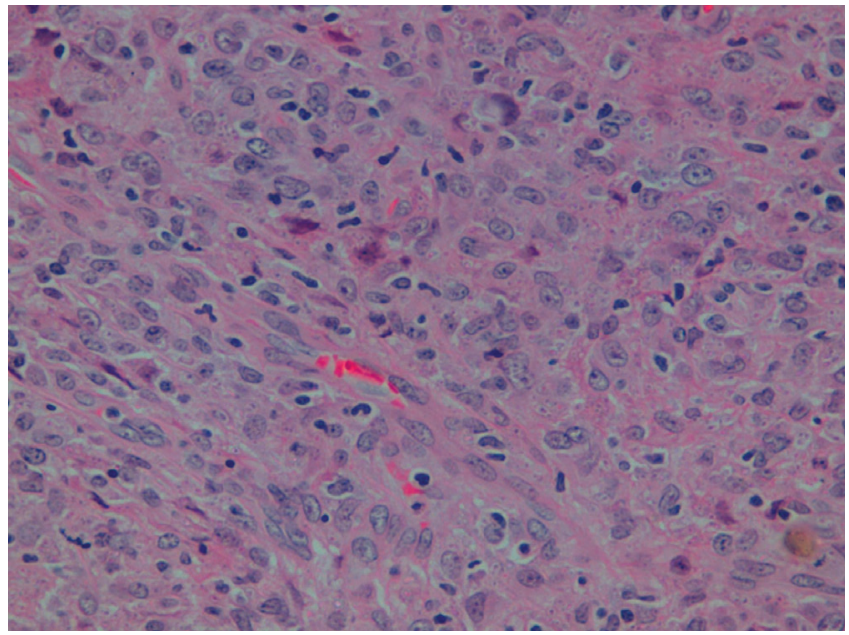

Figura 5 - Exame histológico $(H \& E, 40 x)$ : derme com infiltrado inflamatório granulomatoso com numerosos histiocitos contendo no seu interior estruturas arredondadas pequenas translúcidas sugestivas de corresponderem a Histoplasma capsulatum.

periférico revelaram anemia ligeira: $\mathrm{Hg} 10,2 \mathrm{~g} / \mathrm{dL}$, velocidade de hemossedimentação de $100 \mathrm{~mm} / \mathrm{H}$, VDRL negativo. A função renal, hepática, glicemia, electrólitos, hemograma estavam dentro dos parâmetros normais. A pesquisa de antígeno de criptococos sérico foi negativo.

Foi medicada com anfotericina B durante 21 dias e anti-retroviral (tenofovir, lamivudina e efavirenz), evoluiu com

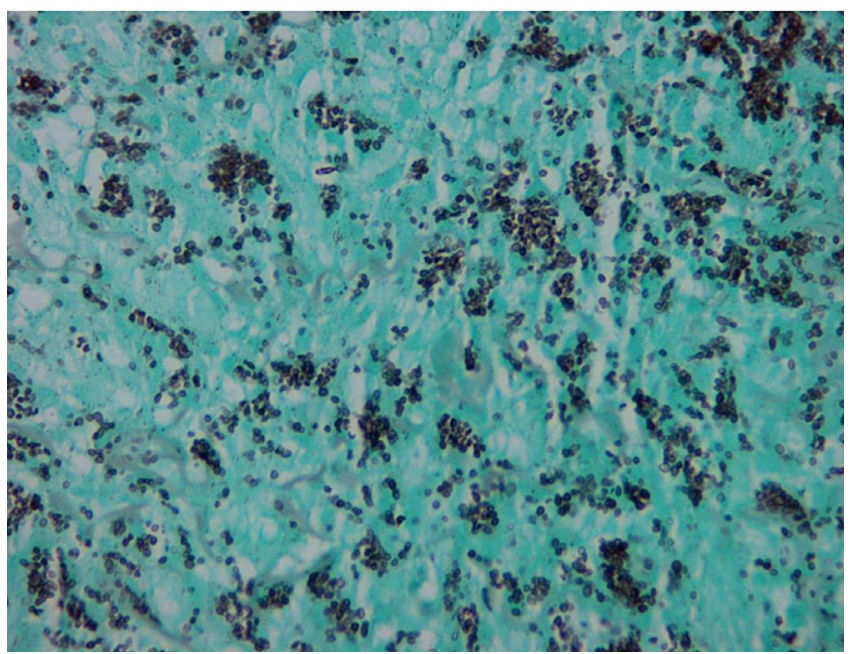

Figura 6 - Exame histológico (Grocott, 40x): Observam-se estruturas arredondadas de cor esverdeada típicas de Histoplasma capsulatum.

melhoria clínica (redução do tamanho da lesões, reepitelização da erosões e melhoria do estado geral), com CD4 3 meses depois de $3 \mathrm{cel} / \mathrm{mm}^{3}$. Teve alta medicada com fluconazol $400 \mathrm{mg} /$ dia e tratamento anti-retroviral. Faleceu 13 meses depois, após gastrenterite aguda cuja causa não foi esclarecida.

\section{DISCUSSÃO}

Embora a histoplasmose cutânea seja frequente em imunodeprimidos, o diagnóstico clínico é difícil pela sua semelhança com outras doenças infecciosas que podem afectar a pele, tais como a criptococose, tuberculose, leishmaniose e sífilis, infecções que embora potencialmente sistémicas podem afectar a pele isoladamente. Outros diagnósticos diferenciais são a sarcoidose e o molusco contagioso. Distinguem-se através dos exames complementares analíticos, micológicos e histopatológicos. Nestes últimos têm um papel importante as colorações específicas: ácido peridódico de Schiff (PAS) para os fungos, Fontana-Masson para o criptococo, a coloração de Ziehl-Neelsen para o bacilo da tuberculose, Grocott para histoplasma, Giemsa para a leishmania. A histopatologia é útil no diagnóstico precoce principalmente quando a cultura não está disponível como foi o caso da nossa doente.

Relativamente às variedades capsulatum ou duboisii distinguem-se histologicamente pelo diâmetro. O Histoplasma capsulatum var. duboisii é maior em relação a var. capsulatum, medindo 10-15 $\mu \mathrm{m}$ de diâmetro. As duas variantes de histoplasmose diferem também na apresentação clínica. A variante capsulatum (mundial) cursa geralmente com poucas lesões cutâneas e quando presentes associam-se geralmente a uma infecção sistémica grave. A variedade duboisii (africana) costuma afectar a pele e os ossos. ${ }^{5}$

Não dispomos de radiografia óssea mas destacamos a ausência de queixas a esse nível. Ficamos pois na dúvida sobre a variedade de histoplasma da nossa doente.

O tratamento faz-se em três (3) fases: A primeira com anfotericina $B$ desoxicolato 0,7 a $1 \mathrm{mg} / \mathrm{kg} /$ dia durante pelo menos duas semanas. A segunda fase (consolidação) com itraconazol $200 \mathrm{mg}$ duas vezes ao dia por pelo menos 12 meses e terceira fase (manutenção ou profilaxia secundária) com itraconazol $200 \mathrm{mg} /$ dia. O cetoconazol também pode ser administrado como um tratamento de primeira linha na dose de $600-800 \mathrm{mg} /$ dia por três meses seguido de $400 \mathrm{mg} /$ dia por 6-12 meses e às vezes por mais tempo. O fluconazole é relatado como eficaz em apenas alguns casos.

Deve ser descontinuado após o período mínimo de um (1) ano de ausência de sintomas e CD4+ maior do que $150 / 200$ células $/ \mathrm{mm}^{3}$ por mais de seis meses.

O curso da doença é progressiva e fatal quando há envolvimento visceral. ${ }^{5}$ Baixa contagem de CD4 $(<50 /$ mm3) e ausência de tratamento antiretroviral são factor preditivos de mau prognóstico. ${ }^{6}$

O caso da nossa doente teve um desenlace fatal após aparente melhoria clínica. Para esse facto terá contribuído o facto de estar gravemente imunodeprimida na altura do diagnóstico embora não se tivesse comprovado envolvimento sistémico por histoplasma. A gastrenterite também não foi estudada pelo que não sabemos se teve relação com esta infecção. 


\section{Caso Clínico}

Conflitos de interesse: Os autores declaram não possuir conflitos de interesse.

Suporte financeiro: $O$ presente trabalho não foi suportado por nenhum subsídio ou bolsa.

Confidencialidade dos dados: Os autores declaram ter seguido os protocolos do seu centro de trabalho acerca da publicação dos dados de doentes.

Protecção de pessoas e animais: Os autores declaram que os procedimentos seguidos estavam de acordo com os regulamentos estabelecidos pelos responsáveis da Comissão de Investigação Clínica e Ética e de acordo com a Declaração de Helsínquia da Associação Médica Mundial.

Consentimento dos Doentes: Obtido.

Conflicts of interest: The authors have no conflicts of interest to declare.

Financing Support: This work has not received any contribution, grant or scholarship.

Confidentiality of data: The authors declare that they have followed the protocols of their work center on the publication of data from patients.

Protection of human and animal subjects: The authors declare that the procedures followed were in accordance with the regulations of the relevant clinical research ethics committee and with those of the Code of Ethics of the World Medical Association (Declaration of Helsinki).

Patients consent: Obtained.

\section{REFERÊNCIAS}

1. Ferreira MS, Borges A. Histoplasmosis. Rev Soc Bras Trop. 2009; 42:680-2.

2. Saheki MN, Schubach Ade O, Salgueiro Mde M, Conceição-Silva F, Wanke B, Lazera M. Histoplasmose cutanea primaria: relato de caso em paciente imunocompetente e revisao de literatura. Rev Soc Bras Med Trop. 2008;41:680-2.

3. Gumaa SA, Ahmed MA, Hassan ME, Hassan AM. A case of African histoplasmosis from Sudan. Trans R Soc Trop Med Hyg. 1988;82:503-5.

4. Bahr NC, Antinori S, Wheat LJ, Sarosi GA. Histoplasmosis infections worldwide: thinking outside of the Ohio River valley. Curr Trop Med Rep. 2015;2:70-80.

5. Ravindran S, Sobhanakumari K, Celine M, Palakkal S. African histoplasmosis: the first report of an indigenous case in India. Int J Dermatol. 2015;54:451-5.

6. Nacher M, Adenis A, Blanchet D, Vantilcke V, Demar $M$, Basurko $C$, et al. Risk factors for disseminated histoplasmosis in a cohort of HIV-infected patients in French Guiana. PLoS Negl Trop Dis. 2014;8:e2638.

7. Guarner J, Brandt ME. Histopathologic diagnosis of fungal infections in the 21 st century. Clin Microbiol Rev. $2011 ; 24: 247-80$.

8. Wolff K, Goldsmith L, Katz S, Gilchrest B, Paller AS, Leffell D. Fitzpatrick's Dermatology in General Medicine. 7th ed. New York: McGraw-Hill; 2008.

9. Bolognia JL, Jorizzo JJ, Schaffer JV, Callen JP, Cerroni L, Heymann WR, et al. 3rd ed. London:Elsevier; 2012.

10. Griffiths C, Barker J, Bleiker T, Chalmers R, Creamer D. Rooks Textbook of Dermatology. 8th ed. London: Wiley; 2016. 